\title{
Evaluation for Vesicoureteric Reflux Following Febrile Urinary Tract Infections
}

\author{
Jitendra Meena $^{1} \cdot$ Aditi Sinha $^{1}$ \\ Received: 24 June 2019 / Accepted: 25 June 2019 / Published online: 6 July 2019 \\ (C) Dr. K C Chaudhuri Foundation 2019
}

Urinary tract infections (UTI), among the most common infections in childhood, are important to recognize and treat because pyelonephritis carries a risk of permanent renal injury. Parenchymal scars are observed in 10-15\% of patients with first episode of UTI [1]. Since vesicoureteral reflux (VUR) and bladder bowel dysfunction (BBD) are important risk factors for recurrence of UTI, evaluation following an episode of UTI focuses on detecting such 'at-risk' patients. Based on the performance and timing of ultrasonography, voiding cystourethrogram (VCUG) and dimercaptosuccinic acid (DMSA) scan, two approaches to evaluation are conventionally recognized [2]. While ultrasonography rules out significant structural malformations and gross pelvicalyceal or ureteric dilatation, it has low sensitivity for VUR. Hence, the 'bottom-up' approach, which intends to detect all VUR, relies on performing VCUG, followed by DMSA in those with VUR. In contrast, the 'top-down' approach recommends ultrasonography and DMSA during acute infection, limiting performance of VCUG to patients in whom DMSA suggests pyelonephritis. While the former approach is more often feasible, cost-effective and sensitive, it involves the discomfort associated with urinary catheterization. On the other hand, the 'top-down' approach is limited by cost and lack of availability of DMSA, significant inter-observer variability in its reporting, higher exposure to radiation than with VCUG, and low sensitivity in detection of VUR [2]. Diagnosis of VUR by direct radionuclide cystography, magnetic resonance urography or contrast-enhanced voiding cysto-ultrasonography, while promising, is yet to find application in current algorithms [2].

The study by Balestracci et al., published in this issue of the Journal, evaluated the efficacy of a modified 'top-down'

Aditi Sinha

aditisinhaaiims@gmail.com

1 Division of Nephrology, Department of Pediatrics, All India Institute of Medical Sciences, New Delhi 110029, India approach [3]. Similar to the recommendations of the Indian Society of Pediatric Nephrology [4], they delayed DMSA to detect scarring as a consequence of pyelonephritis, and propose it as an alternative to VCUG in detecting high-grade VUR. Retrospective review of records, for children older than $3 \mathrm{y}$ who underwent all three procedures during evaluation of UTI, found that an abnormal delayed DMSA scan, observed in $57.6 \%$ patients, predicted all grades of VUR with $93 \%$ sensitivity and $75 \%$ specificity. An abnormal DMSA scan was strongly associated with high-grade VUR; all patients with the latter had abnormal DMSA [3]. Compared to these results, delayed DMSA had satisfactory but lower sensitivity ( $83 \%$ and $87 \%$ vs. $93 \%$ ) in detecting high-grade VUR in two studies $[5,6]$. Selection bias might plausibly explain the strength of association in the present report, as patients with high-grade VUR, and therefore, those at risk of scarring, are the ones most likely to have undergone all three investigations. The high proportion of girls in the present study, as compared to previous reports $[5,6]$, might have altered the prevalence of abnormal scintigraphy. Further, as the scan was delayed by almost one year from the occurrence of the first febrile UTI, a proportion of patients, particularly those with high grade VUR, might have had another episode of UTI in the interim, thus increasing the prevalence of, and strengthening the association of VUR with, scarring.

Delayed DMSA scan is expected to be less sensitive and more specific than acute phase scintigraphy in detecting highgrade VUR, as the former detects permanent parenchymal injuries that are expected to be more frequent in this category of patients. A systematic review by the Cochrane Renal Group reported satisfactory sensitivity $(93 \%)$ but moderate specificity $(44 \%)$ for the acute DMSA scan in identifying high-grade VUR [7]. However, enthusiasm in advocating DMSA universally for all children with their first febrile UTI should be tempered by considerations listed previously. While delayed DMSA scan seems useful in screening for high grade VUR in patients presenting late after a febrile UTI, delaying DMSA for several months while deferring VCUG and interventions 
for prevention of UTI in patients with potentially high-grade VUR might not be prudent and risks further infections. Further, delayed DMSA is unlikely to detect all patients with high-grade VUR, as only $10-15 \%$ of patients presenting with their first febrile UTI will develop scars.

The primary aim when managing patients with VUR is the prevention of UTI, while aspiring to reduce renal scarring. Antibiotic prophylaxis seems to be effective in reducing risk of UTI primarily in patients with high-grade VUR [8]. Importantly, evidence from controlled studies, including the large multicentric RIVUR trial, fails to support a role for currently available interventions in reducing the risk of renal scarring, and it is likely that scarring has other determinants, such as BBD and in utero dysplasia. Hence, the approach to evaluating patients following UTI is increasingly becoming more conservative. Future approaches might show increased emphasis on risk stratification, and reduced focus on detection of any, rather than high grade, VUR, potentially using less invasive strategies with low radiation exposure.

\section{Compliance with Ethical Standards}

Conflict of Interest None.

\section{References}

1. Shaikh N, Ewing AL, Bhatnagar S, Hoberman A. Risk of renal scarring in children with a first urinary tract infection: a systematic review. Pediatrics. 2010;126:1084-91.

2. Routh JC, Grant FD, Kokorowski PJ, et al. Economic and radiation costs of initial imaging approaches after a child's first febrile urinary tract infection. Clin Pediatr. 2012;51:23-30.

3. Balestracci A, Montecuco M, Serviddio C, et al. Role of late DMSA renal scan in detecting high-grade vesicoureteral reflux. Indian $\mathrm{J}$ Pediatr. 2019. https://doi.org/10.1007/s12098-019-02917-4.

4. Indian Society of Pediatric Nephrology. Revised statement on management of urinary tract infections. Indian Pediatr. 2011;48:709-17.

5. Wongbencharat K, Tongpenyai Y, Na-Rungsri K. Renal ultrasound and DMSA screening for high-grade vesicoureteral reflux. Pediatr Int. 2016;58:214-8.

6. Quirino IG, Silva JM, Diniz JS, et al. Combined use of late phase dimercapto-succinic acid renal scintigraphy and ultrasound as first line screening after urinary tract infection in children. J Urol. 2011;185:258-63.

7. Shaikh N, Spingarn RB, Hum SW. Dimercaptosuccinic acid scan or ultrasound in screening for vesicoureteral reflux among children with urinary tract infections. Cochrane Database Syst Rev. 2016;7: CD010657.

8. Wang ZT, Wehbi E, Alam Y, Khoury A. A reanalysis of the RIVUR trial using a risk classification system. J Urol. 2018;199:1608-14.

Publisher's Note Springer Nature remains neutral with regard to jurisdictional claims in published maps and institutional affiliations. 